\title{
Influence of Boundary Conditions on the Dynamic Characteristics of Squeeze Films in MEMS Devices
}

\author{
Ashok Kumar Pandey, Rudra Pratap, Member, IEEE, and Fook Siong Chau
}

\begin{abstract}
Micromechanical structures that have squeeze-film damping as the dominant energy dissipation mechanism are of interest in this paper. For such structures with narrow air gap, the Reynolds equation is used for calculating squeeze-film damping, which is generally solved with trivial pressure boundary conditions on the side walls. This procedure, however, fails to give satisfactory results for structures under two important conditions: 1) for an air gap thickness comparable to the lateral dimensions of the microstructure and 2) for nontrivial pressure boundary conditions such as fully open boundaries on an extended substrate or partially blocked boundaries that provide side clearance to the fluid flow. Several formulas exist to account for simple boundary conditions. In practice, however, there are many micromechanical structures such as torsional microelectromechanical system (MEMS) structures that have nontrivial boundary conditions arising from partially blocked boundaries. Such boundaries usually have clearance parameters that can vary due to fabrication. These parameters, however, can also be used as design parameters if we understand their role on the dynamics of the structure. We take a MEMS torsion mirror as an example device that has large air gap and partially blocked boundaries due to static frames. We actuate the device and experimentally determine the quality factor $Q$ from the response measurements. Next, we model the same structure in ANSYS and carry out computational fluid dynamics analysis to evaluate the stiffness constant $K$, the damping constant $D$, and the quality factor $Q$ due to the squeeze film. We compare the computational results with experimental results and show that without taking care of the partially blocked boundaries properly in the computational model, we get unacceptably large errors.

[2006-0209]
\end{abstract}

Index Terms-Computational fluid dynamics (CFD), experimental quality factor, microelectromechanical system (MEMS), partially blocked boundaries, squeeze-film damping, torsional motion.

\section{INTRODUCTION}

$\mathbf{E}$ VALUATION of fluid damping and spring characteristics is critical in the design and analysis of dynamic microelectromechanical system (MEMS) devices where the motion

Manuscript received September 26, 2006; revised May 1, 2007. This work was supported in part by the Department of Science and Technology (DST), New Delhi, India, and in part by the National University of Singapore, Singapore. Subject Editor N. Aluru.

A. K. Pandey and R. Pratap are with the Department of Mechanical Engineering, Indian Institute of Science, Bangalore 560 012, India (e-mail: ashok@ mecheng.iisc.ernet.in; pratap@ mecheng.iisc.ernet.in).

F. S. Chau is with the Department of Mechanical Engineering, National University of Singapore, Singapore 119260 (e-mail: mpecfs@nus.edu.sg).

Color versions of one or more of the figures in this paper are available online at http://ieeexplore.ieee.org.

Digital Object Identifier 10.1109/JMEMS.2007.901135 of the structure is in a fluid medium such as air or some other gas. For MEMS devices such as a torsional mirror or an accelerometer, in which the main sensing/actuating element executes transverse motion with respect to a fixed substrate, fluid damping is mainly due to the squeeze film and drag on the lateral and top surfaces [1], [2].

The squeeze-film damping in MEMS devices is generally calculated by solving the conventional Reynolds equation. The analytical results for the squeeze-film damping and spring forces in a system with a plate executing small amplitude harmonic motion are given in [3], and the corresponding torques in the case of torsional motion are derived in [4]. These damping and spring forces in complex structures are also calculated by solving Reynolds equation numerically for rigid [5] and flexible structures [6] executing transverse motion. Since the computation methods are intense and time consuming, Hung et al. [7], [8] have proposed a low-order model for fast dynamical simulation by extracting basis functions from a few finite-element method (FEM) simulation results. In all these studies, the Reynolds equation is solved with trivial pressure boundary condition $(P=0)$ on the free edges and trivial flow conditions $(V=0)$ on the fixed edges.

Although the trivial pressure boundary condition hardly represents the actual condition around a moving structure, it is helpful in predicting the nature of squeeze-film effects for structures with large length to air gap ratio. For comparable length to air gap ratio, the effect of surrounding pressure on the squeeze-film damping can be significant. Thus, a nontrivial boundary condition should be used while calculating the effect of the squeeze film. There are a few studies ([9]-[12]) that present various ways to consider nontrivial boundary conditions on squeeze-film damping under various conditions. To apply a nontrivial pressure boundary condition on the plate edges, Vemuri et al. [9] have performed numerical simulation on a 3-D domain by extending the control volume beyond the edges of the oscillating plate. The extension to the control volume is taken such that it is greater than the distance from the plate edges where the pressure difference becomes zero. Subsequently, the extended length and width of the plate are found by comparing the numerical solution with the analytical damping and spring constants obtained using the trivial boundary condition for the effective plate with elongated length and width. The required extension to the plate dimensions is a function of the characteristic dimension of the squeeze film, i.e., the characteristic length to air gap ratio, and the frequency of operation. Veijola et al. [10] have proposed two 


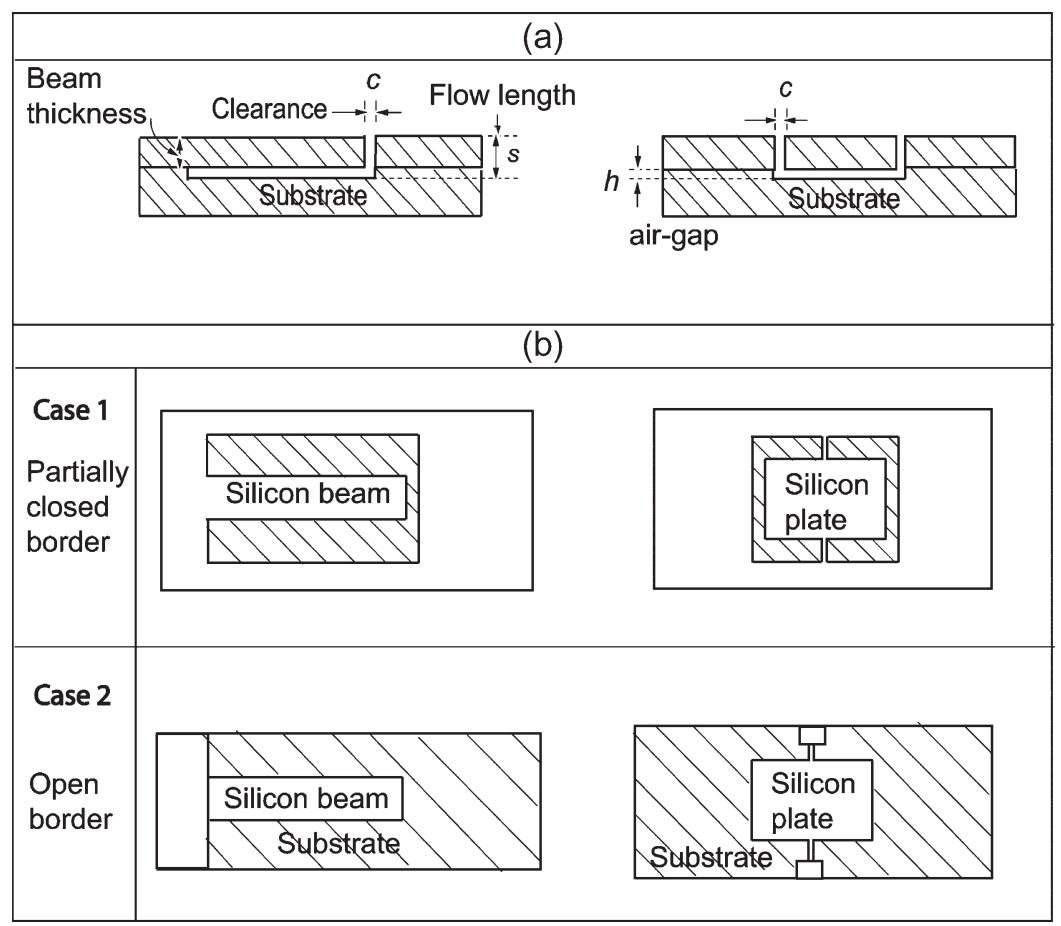

Fig. 1. (a) Side view and (b) top view of the microfabricated cantilever beam and torsional structure are shown with the partially blocked boundaries.

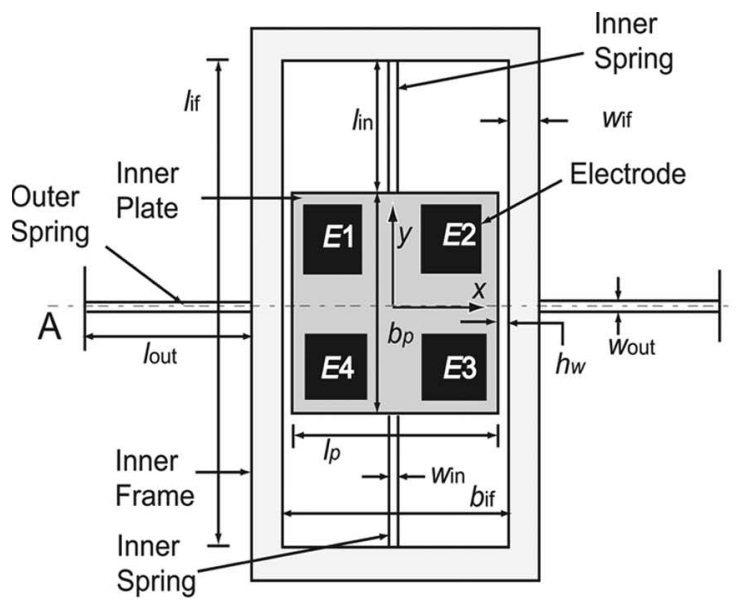

(a)

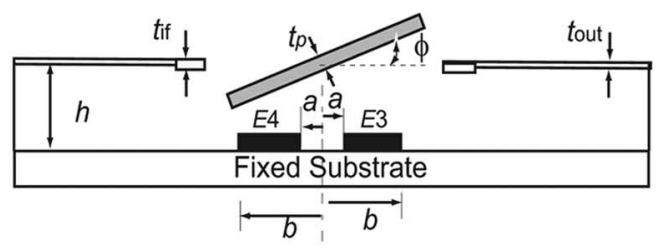

(b) A-A' view

\section{Boundary Conditions}

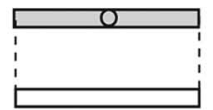

(c)

$A^{\prime}$

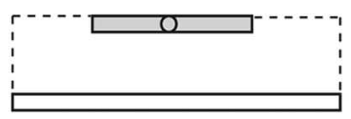

(d)

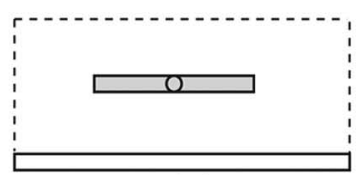

(e)

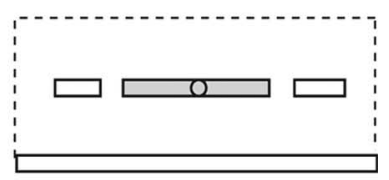

(f)

Fig. 2. Schematic diagram of the (a) double-gimballed torsion mirror and (b) its sectional view along A-A'. To evaluate the effect of different factors on fluid damping in the structure shown in (a) and (b), an appropriate boundary condition is used. The extended boundaries (shown dotted) are taken in four different ways to capture different effects: (c) squeeze-film effect; (d) squeeze-film effect, end effect, and drag effect only on the lateral side of the vibrating structure; (e) squeeze-film effect, end effect, and drag effect on the side and the top of the oscillating structure; and (f) in addition to the effects captured in (e), the effect of a static frame separated by a small distance from the boundaries of the oscillating structure is also considered.

models to consider the effect of open boundaries on squeezefilm damping: 1) the border flow channel model where imaginary flow channels are used at the edges of the plate; and 2) the surface extension model where the edges are extended to accommodate the effect of open borders. They have calculated the appropriate extension to the original dimensions of the plate by comparing the numerical squeeze-film resistance obtained using trivial pressure boundary conditions on the extended 
volume with the analytical resistance value for the extended plate. They have found that in the continuum flow regime, the validity of Reynolds equation can be extended to systems with characteristic structural dimensions comparable to the air gap thickness if the structural dimensions are increased by 1.3 times the air gap thickness. The model is also modified to include the rarefaction effect in the slip flow regimes. In torsional motion, the maximum relative error under this condition is found to be less than $10 \%$ for the length-to-air-gap ratio greater than 10. Since the dimensional extension is obtained under the assumption of incompressible flow, the model is reported to be valid below $500 \mathrm{kHz}$. The above studies are mainly done for fully open boundaries, as shown in case 2 of Fig. 1(b). In order to model the effect of partially blocked boundaries, where the fluid flows through a narrow opening on the side of the plate, as shown in case 1 of Fig. 1(b), Darling et al. [12] have proposed a compact model under arbitrary venting conditions by solving acoustic equations. They have obtained an analytical solution for the squeeze-film damping and spring forces by solving the conventional Reynolds equation for trivial as well as nontrivial boundary conditions. In order to consider the effect of side walls, Hao et al. [11] have obtained the squeeze-film damping and spring torques for torsional motion by taking trivial pressure boundary conditions on the side walls rather than on the edges of the oscillating plate. However, the zero-pressure boundary condition on the side walls is not a good idea when the side walls are placed closer to the oscillating plate. The effect of nontrivial boundary conditions in partially blocked boundaries requires thorough investigation in order to find the distance from the edges of the oscillating structure where the pressure difference becomes zero. The models presented in the last two references [1], [12] are restricted by the upper frequency limit below which inertial effects are negligible. Also, these models do not apply to cases with large air gaps (where the Reynolds equation is not applicable due to the violation of its basic assumption of small air gap to length ratio). These studies are also not applicable to structures with complex boundary conditions such as the partially blocked boundaries that exist in the MEMS device under consideration [which is shown in Fig. 2(a)]. Partially blocked boundaries have clearance parameters that can have small enough values to be significantly affected by fabrication processes. The gap between a side wall (a fixed boundary) and a vibrating structure is one such parameter. The geometric parameters that characterize the fluid domain between the fixed boundaries and the moving structure are of interest here. These parameters can also be used as effective design parameters if we understand their role on the dynamics of the structure.

In this paper, we take an example structure with partially blocked boundaries and carry out computational fluid dynamics (CFD) simulations using several models for boundary conditions to evaluate the dynamic characteristics of the structure. In particular, we compute the quality factor with each of the boundary models and compare the results with experimentally obtained values. We also compare the numerical and experimental results with the available analytical formulas and discuss the validity of analytical formulas under different conditions.

\section{THEORY}

\section{A. Governing Equations}

In a system where a structure vibrates near a fixed substrate in a fluid medium, the fluid behavior can be modeled using the Navier-Stokes equation [14], [15]. For a low vibration frequency, assumptions of incompressibility and laminar flow hold good. However, as the excitation frequency increases, compressibility and inertial effects become significant. Under isothermal flow conditions and constant dynamic viscosity, we get the following set of equations for fluid flow [14], which are solved using ANSYS:

$$
\begin{gathered}
\frac{\partial \rho}{\partial t}+\nabla \cdot(\rho \vec{u})=0 \\
\rho \frac{D \vec{u}}{D t}=\rho \vec{g}-\nabla p+\mu \nabla^{2} \vec{u} \\
\rho \propto p
\end{gathered}
$$

where $\vec{u}$ is the fluid velocity, $p$ is the fluid pressure, $\rho$ is the fluid density, $\vec{g}$ is the acceleration due to gravity, and $\mu$ is the dynamic viscosity. In problems related to MEMS devices, the effect of body forces such as $\rho \vec{g}$ can be neglected without any substantial error. The time rate of change of density can be expressed in terms of the time rate of change of pressure as $\partial \rho / \partial t=(\partial \rho / \partial p)(\partial p / \partial t)$, where $\partial \rho / \partial p=\rho / \kappa$, and $\kappa$ is the bulk modulus of the fluid. In this paper, the fluid considered is air. For air, at temperature $T=298 \mathrm{~K}$ and pressure $p_{a}=$ $1.013 \times 10^{5} \mathrm{~Pa}, \mu \approx 1.8 \times 10^{-5} \mathrm{~N}-\mathrm{s} / \mathrm{m}^{2}, \rho_{a}=1.2 \mathrm{~kg} / \mathrm{m}^{3}$, and the isothermal bulk modulus $\kappa_{T}=p_{a}=1.013 \times 10^{5} \mathrm{~Pa}$. We must add here that at sufficiently high frequencies or velocities, adiabatic conditions may have to be used in place of isothermal conditions.

\section{B. Boundary Conditions}

Based on the type of opening on the side of the vibrating structure, the boundary conditions can be mainly of the following three kinds.

1) Fully closed boundaries: In this case, the fluid remains inside the cavity. For pure transverse motion, the fluid behaves like a spring, while for torsional motion, there is also some loss due to the lateral fluid motion in the air gap. The magnitude of this effect depends on the frequency of excitation [12]. Under this condition, the fluid velocity along the boundaries is zero.

2) Fully open boundaries: In this case, the fluid can flow freely from the boundaries, and the pressure variation is taken as zero along the boundaries, i.e., $\Delta p=0$. However, because of the sudden flow expansion from the closed cavity to the open surroundings, the pressure on the boundary is different from the ambient pressure. This difference in pressure is called the open border effect or the end effect [17]. This effect is negligible for a system with small air gap $h_{0}$ to length $L$ (or width $W$ ) ratio. Under this condition, trivial pressure boundary conditions can be used to calculate damping. For incompressible 


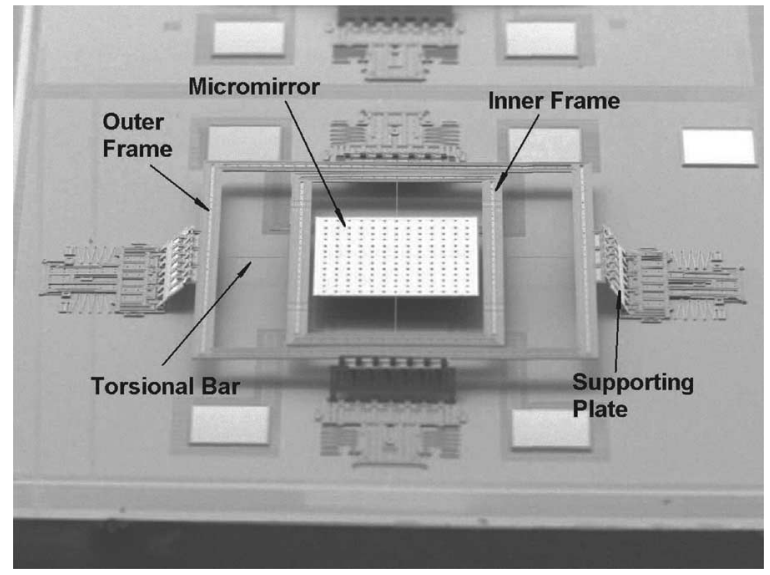

Fig. 3. Picture of double-gimballed MEMS torsion mirror.

flow, the damping constant under this condition is given by [4]

$$
C_{\phi}=\frac{192 W L^{5} \mu}{\pi^{6} h_{0}^{3}} \sum_{\text {even odd }}^{m} \sum_{(m n)^{2}\left(m^{2}+n^{2}\left(\frac{L}{W}\right)^{2}\right)}^{n}
$$

where $\mu$ is the fluid viscosity. For comparable air gap and structural length, the open border effect can be modeled by applying either a nontrivial boundary condition on the original boundaries or a trivial boundary condition on the extended boundaries of the oscillating structure. For low-frequency torsional motion about an axis bisecting the plate length, Veijola et al. [10] have proposed the extension of the original length $l$ of the oscillating plate by $1.3 h_{0}$, i.e., $l_{\text {eff }}=l+1.3 h_{0}$, where $h_{0}$ is the initial air gap thickness. Consequently, for an air gap to length ratio of $\sim 1$ in a structure with fully open boundaries, (4) can be used to calculate damping by replacing $L$ and $W$ with $L_{\text {eff }}=L+1.3 h_{0}$ and $W_{\text {eff }}=W+1.3 h_{0}$. After calculating the damping force in the two different cases, the value of the moment of inertia $I$ and the angular velocity $\omega$ of the original structure are used to calculate the quality factor from

$$
Q_{\text {anal }}=\frac{I \omega}{C_{\phi}} .
$$

3) Partially open boundaries: Unlike the previous cases, the boundaries are neither completely open nor completely closed. In this case, $\alpha p+\beta \nabla p=0$ is used on the boundaries, where $\alpha$ and $\beta$ are constants whose values depend on the closed and open portions of the boundaries.

\section{Solution Methodology}

To study the effect of boundaries on the dynamic characteristics of the squeeze film, we choose a MEMS torsion mirror (shown in Fig. 3) as a test structure whose dimensions are given in Table I. Our methodology involves experimental and numerical investigations that include different boundary effects. In this section, we outline the procedure we have followed for these studies.
1) Experimental Procedure: In order to evaluate the quality factor of the torsional microstructure under ambient conditions, we build a test setup shown schematically in Fig. 4. The dynamics of the torsional structure involves rotational vibration of the inner plate about the inner torsional bars. The experimental studies in this case require a function generator, an oscilloscope, two dc power sources, a LASER source, a LASER pointer, a position sensing device (PSD), a platform, and three adjustable stages to mount the PSD, LASER pointer, and test structure, respectively. The procedure of determining the quality factor experimentally involves the following steps.

1) An input voltage $V=V_{\text {input }} \sin 2 \pi f t$, where $f$ is the input frequency, and $t$ is the time, and a bias voltage $V_{\text {bias }}$ are applied to the electrodes $\left(E_{1}\right.$ and $\left.E_{4}\right)$ and the inner plate [see Fig. 2(a)], respectively. Here, $V_{\text {bias }}=20 \mathrm{~V}$, and $V_{\text {input }}=0.5 \mathrm{~V}$. The static angular deflection due to the applied bias voltage is about $5 \times 10^{-3} \mathrm{rad}$, which is negligibly small. The application of a sinusoidal voltage results in angular oscillation $\phi$ of the inner plate about the inner torsional bars.

2) A LASER source and a LASER pointer are used to focus the LASER beam at a point on the top of the vibrating plate. After getting reflected from the plate, the LASER beam falls on the PSD, as shown in Fig. 5(a).

3) As the plate vibrates, the voltage reading of the PSD is taken from an oscilloscope. At steady state, the voltage reading is taken, which in turn gives the angular displacement of the oscillating plate from the relation $\phi=\left(V_{p} / d\right)$ (in rad). Here, $V_{p}$ is the PSD output voltage (in the present case, the relation between voltage and displacement is set as $1 \mathrm{~V} \approx 1 \mathrm{~mm}$ ), and $d$ (in millimeters) is the center distance between the PSD and the LASER spot on the vibrating plate.

4) By repeating steps $1-3$ for different frequencies and by reading the corresponding maximum angular displacements, we get the frequency response curve as shown in Fig. 5.

5) Using the half-width method [20], we measure the quality factor $Q_{\text {exp }}$ from the frequency response curve.

The expression for quality factor is given by

$$
Q_{\exp } \approx \frac{1}{2 \xi}=\frac{f_{d}}{f_{2}-f_{1}}
$$

where $f_{1}$ and $f_{2}$ are the half power frequencies.

By following the above experimental procedure, we get the response curve for the angular oscillation of the inner plate, as shown in Fig. 5. The experiment is repeated ten times, and the average of all data points is taken. From the response curve, we get the quality factor $Q_{\exp } \approx 9.19$ and the damping factor $\xi=0.054$ from $f_{1}=500.4 \mathrm{~Hz}$ and $f_{2}=558 \mathrm{~Hz}$. The damped natural frequency comes out to be $f_{d}=529.2 \mathrm{~Hz}$. The maximum angular displacement at resonant frequency is found to be $0.035 \mathrm{rad}$.

2) Numerical Procedure: The CFD procedure to calculate the dynamic characteristics of the squeeze film under different boundary conditions involves three major steps: 1$)$ modeling and discretizing the fluid domain; 2) solving for the pressure 
TABLE I

Dimensions of MEMS TORSION MirRor SHOwn In FIG. 2

\begin{tabular}{|c|c|c|}
\hline Symbol & Description & Values \\
\hline$l_{p}$ & length of the inner plate & $400 \mu \mathrm{m}$ \\
\hline$b_{p}$ & width of the inner plate & $400 \mu \mathrm{m}$ \\
\hline$t_{p}$ & thickness of the inner plate & $4.25 \mu \mathrm{m}$ \\
\hline$l_{\text {if }}$ & length of the inner frame & $778 \mu \mathrm{m}$ \\
\hline$b_{\text {if }}$ & breadth of the inner frame & $438 \mu \mathrm{m}$ \\
\hline$w_{\text {if }}$ & width of the inner frame & $52 \mu \mathrm{m}$ \\
\hline$t_{\text {if }}$ & thickness of the inner frame & $3.5 \mu \mathrm{m}$ \\
\hline$h_{w}$ & gap between the inner plate and the inner frame & $19 \mu \mathrm{m}$ \\
\hline$l_{\text {in }}$ & length of the inner torsional bar & $180 \mu \mathrm{m}$ \\
\hline$l_{\text {out }}$ & length of the outer torsional bar & $180 \mu \mathrm{m}$ \\
\hline$w_{\text {in }}$ & width of the inner torsional bar & $2 \mu \mathrm{m}$ \\
\hline$w_{\text {out }}$ & width of the outer torsional bar & $2 \mu \mathrm{m}$ \\
\hline$t_{\text {in }}$ & thickness of the inner torsional bar & $1.5 \mu \mathrm{m}$ \\
\hline$t_{\text {out }}$ & thickness of the outer torsional bar & $1.5 \mu \mathrm{m}$ \\
\hline$p_{h}$ & pitch of perforation & $32.5 \mu \mathrm{m}$ \\
\hline$l_{h}$ & size of a single square & $3 \mu \mathrm{m}$ \\
\hline$h_{0}$ & air-gap thickness & $80 \mu \mathrm{m}$ \\
\hline$a$ & inner side of the electrode from the central axis & $10 \mu \mathrm{m}$ \\
\hline$b$ & outer side of the electrode from the central axis & $170 \mu \mathrm{m}$ \\
\hline$\rho_{\text {poly }}$ & density of polysilicon & $2300 \mathrm{~kg} / \mathrm{m}^{3}$ \\
\hline$G$ & & $6.5 \times 10^{10} \mathrm{~Pa}$ \\
\hline
\end{tabular}

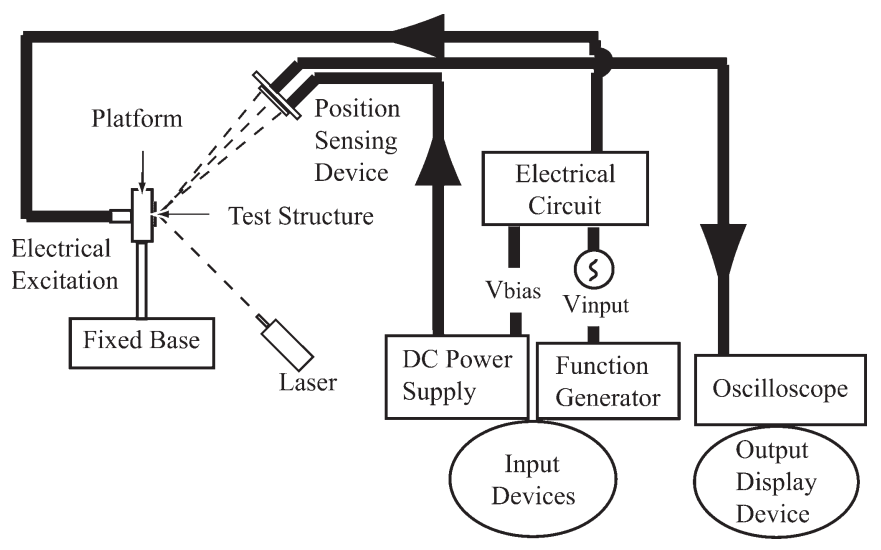

Fig. 4. Outline sketch of the experimental setup.

distribution with the given load and boundary conditions using (1)-(3); and 3) computing the desired forces and other quantities from the computed pressure distribution and velocity field. An outline of the different computational substeps is given in Fig. 11. A few important aspects and expressions related to modeling and estimation of the spring force, damping force, and quality factor are discussed below.

1) Issues related to modeling: First, a 3-D fluid domain around the test structure is created. The outer boundaries, which are shown dotted in Fig. 2(f), are extended from the side and the top surface of the oscillating structure. The extension factor depends on various factors such as the air gap thickness to the length ratio [10] and the

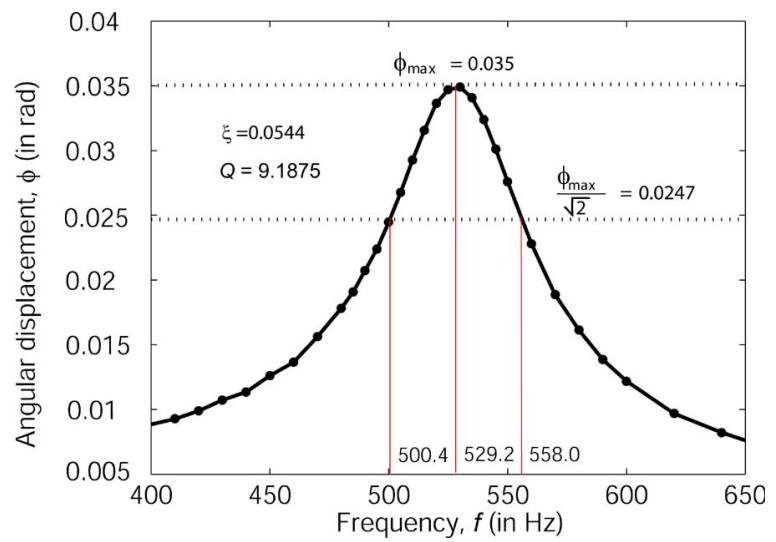

Fig. 5. Frequency response (in terms of angular displacement $\phi$ ) of MEMS torsion mirror vibrating about the $y$-axis for bias voltage $V_{\mathrm{bias}}=20 \mathrm{~V}$ and $\left|V_{\text {input }}\right|=0.5 \mathrm{~V}$.

nature of the boundary condition such as fully or partially blocked boundaries. The reasons for extending the boundaries in the open and partially blocked conditions are mainly twofold: first, to accommodate the effect of sudden expansion and contraction through the side and top openings; and second, to account for the drag forces on the side walls and the top surface of the oscillating structure (see Fig. 2(a) and (b) for the openings around the inner plate). The main criteria for selecting the extension of surfaces is that it should be greater than the distance where the solution converges for pressure distribution 


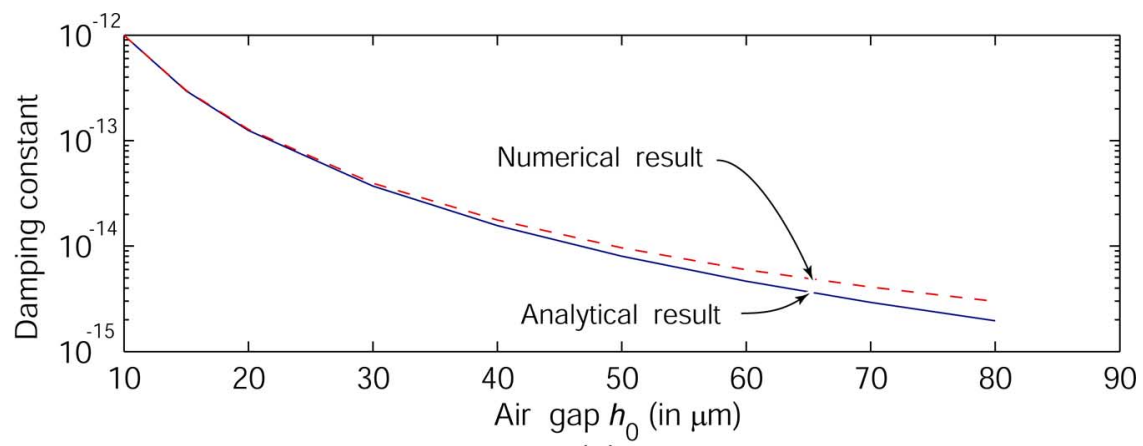

(a)

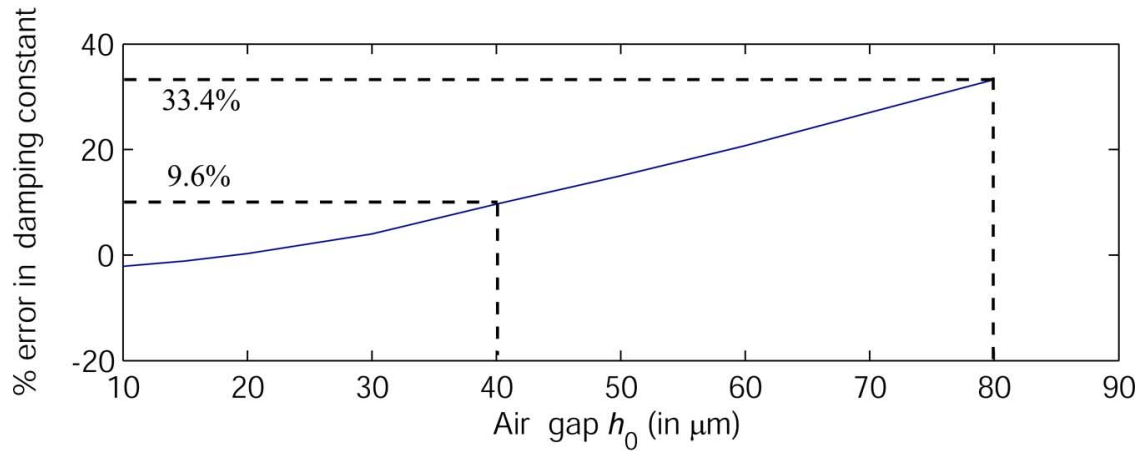

(b)

Fig. 6. (a) Comparison between the numerical and analytical damping constants for different air-gap thicknesses. (b) Percentage error in analytical result with respect to numerical result with different air-gap thickness.

around the structure. For example, the boundaries in Fig. 2(e), which are similar to the condition around the experimental test structure, are extended by $l_{p} / 2$ on the left and right sides of the oscillating plate, and $3 h_{0} / 4$ from the upper surface of the vibrating plate on the top to capture the aforementioned effects, where $l_{p}$ is the length of the plate, and $h_{0}$ is the air-gap thickness.

2) Calculation of the quality factor: After getting the pressure distribution $p$ on the walls of the oscillating plate and integrating the moment of the total back force about the axis of oscillation, we estimate the total back torque on the oscillating structure. Then, after calculating the phase difference $\gamma$ between the total back torque and the angular velocity, we calculate the spring torque, the damping torque, and the quality factor based on the total fluid damping. If the angular displacement of the oscillating structure is $\phi(t)=\Phi \sin \omega t$, where $\omega=2 \pi f=2 \pi / \tau$ is the angular frequency of oscillation, $\tau$ is the time period of oscillation, and $\gamma$ is the phase difference as defined above, then the angular velocity and the total torque can be written as $\dot{\phi}(t)=\Phi \omega \cos \omega t$ and $T=T_{0} \cos (\omega t+\gamma)$, respectively. Under the assumptions of viscous damping and linear spring, the spring and the damping torques are proportional to the angular displacement and the angular velocity, respectively. Therefore, the expressions for the damping and spring torques are given by

$$
\begin{aligned}
& T_{d}(t)=T_{d 0} \cos \omega t \\
& T_{s}(t)=T_{s 0} \sin \omega t
\end{aligned}
$$

where $T_{d 0}=T_{\max } \cos \gamma$, and $T_{s 0}=T_{\max } \sin \gamma$. Thus, the expressions for the damping constant $D$ and the spring constant $K$ are

$$
D=\frac{T_{d 0}}{\Phi \omega} \quad K=\frac{T_{s 0}}{\Phi} .
$$

Now, the quality factor is defined as [20]

$$
Q_{\mathrm{num}}=2 \pi \frac{\frac{1}{2} I \Phi^{2} \omega^{2}}{\int_{0}^{\tau} T_{d}(t) \dot{\phi}(t) d t}=\frac{I \Phi \omega^{2}}{T_{d 0}}=\frac{I \omega}{D}
$$

where $I$ is the moment of inertia about the axis of rotation. For the present structure, as shown in Fig. 2(a) and (b), $I=\rho l_{p} b_{p} t_{p}\left(l_{p}^{2}+t_{p}^{2}\right) / 12$ without perforations, and $\rho l_{p} b_{p} t_{p}\left(l_{p}^{2}+t_{p}^{2}\right) / 12-\sum_{j=1}^{j=n}\left(n \rho l_{h} l_{h} t_{p}\right.$ $\left.\left(l_{h}^{2}+t_{p}^{2}\right) / 12-\sum_{k=-(n+1) / 2}^{k=+(n+1) / 2}\left(\rho l_{h}^{2} t_{p} k^{2} p_{h}^{2}\right)\right)$ with $n \times n$ equally spaced perforations, where $n$ is an odd number and equals 13 in the present case. The $I$ value corresponding to $n=13$ is about $2.106 \times 10^{17} \mathrm{~kg}-\mathrm{m}^{2}$. The net torsional stiffness of the structure is from as $k_{\phi}=\omega^{2} I=\left(2 \pi f_{d}\right)^{2} I=2.3284 \times 10^{-10} \mathrm{~N}-\mathrm{m}$, where $f_{d}=529.2 \mathrm{~Hz}$ is the resonant frequency obtained from Fig. 5.

\section{Results AND Discussion}

First, we discuss the validation of the numerical results by comparing them with the experimental results for the MEMS torsion mirror shown in Fig. 2. We discuss various effects on the dynamic performance of the test structure associated with 


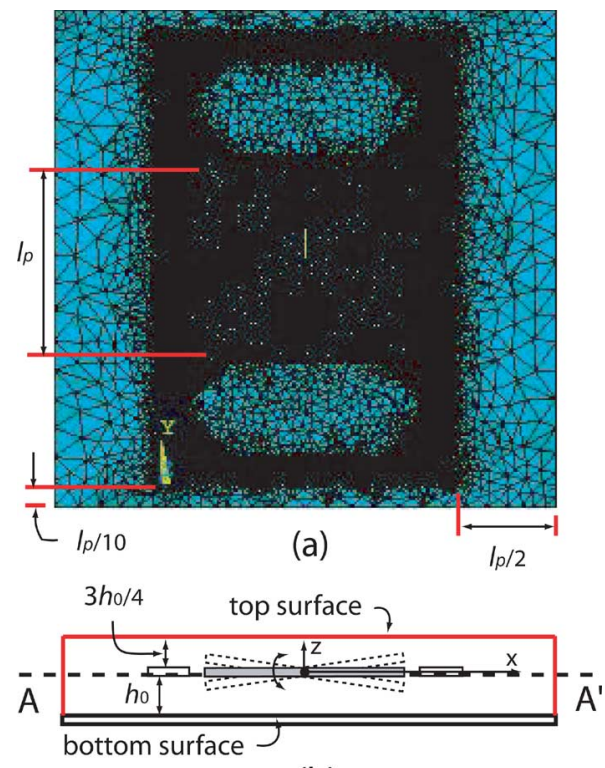

(b)

Fig. 7. (a) Finite elements in the domain bounded by the bottom surface and the section $\mathrm{A}-\mathrm{A}^{\prime}$. (b) Sectional view of the fluid domain passing through the middle of the oscillating plate that is bounded by the top surface, side surfaces, and bottom surface.

different boundary conditions. Before going into the details of numerical modeling, we characterize the nature of the air flow around the given structure using popular characteristic numbers.

1) Modified Reynolds number $\operatorname{Re}=\rho h^{2} \omega / \mu$ : It is a measure of the inertial effects. Using the characteristic flow length $h=h_{0}=80 \mu \mathrm{m}$, the dynamic viscosity of air $\mu=$ $1.8 \times 10^{-5} \mathrm{~N}-\mathrm{s} / \mathrm{m}^{2}$, the density of air $\rho=1.2 \mathrm{~kg} / \mathrm{m}^{3}$, and the angular frequency at resonance $\omega=2 \pi f$, where $f=$ $529.2 \mathrm{~Hz}$, we get $\mathrm{Re}=1.4$. Here, the Reynolds number is greater than 1 because of the large air gap thickness. Under this condition, Reynolds equation, which is used to model the squeeze-film flow, does not hold good. To incorporate the effect of the large air gap thickness, we solve the 3-D Navier-Stokes equation numerically.

2) Squeeze number $\sigma=12 \mu l_{p}^{2} \omega / h_{0}^{2} p_{a}$ : For $l_{p}=400 \mu \mathrm{m}$, $p_{a}=1.013 \times 10^{5} \mathrm{~Pa}$, and the same values of $h_{0}, \omega$, and $\mu$ as mentioned earlier, we get $\sigma=1.77 \times 10^{-4}$. Since $\sigma \ll 1$, the compressibility effect can be neglected.

3) Knudsen number $\mathrm{Kn}=\lambda / h$ : For ambient pressure and temperature, the mean free path $\lambda$ of the air molecules is about $64 \mathrm{~nm}$. For the flow through the air-gap thickness, the characteristic flow length $h=h_{0}$, and $\mathrm{Kn}=8.0 \times$ $10^{-4}$; whereas for the flow through the side opening, $h=h_{w}=19 \mu \mathrm{m}$, and $\mathrm{Kn}=0.003$. Since for both flows $\mathrm{Kn}<0.01$, the fluid flow around the structure is in the continuum flow regime [21]. Therefore, the no-slip boundary conditions at the boundaries of the oscillating structure can be used to solve the Navier-Stokes equation in the fluid domain around the test structure.

4) Perforation ratio $P_{r}=A_{\text {perf }} / A_{\text {plate: }}$ It is the ratio of the perforated area to the plate area. For the perforation area $A_{\text {perf }}=N_{p} \times 3 \mu \mathrm{m} \times 3 \mu \mathrm{m}$, where $N_{p}=13 \times 13$

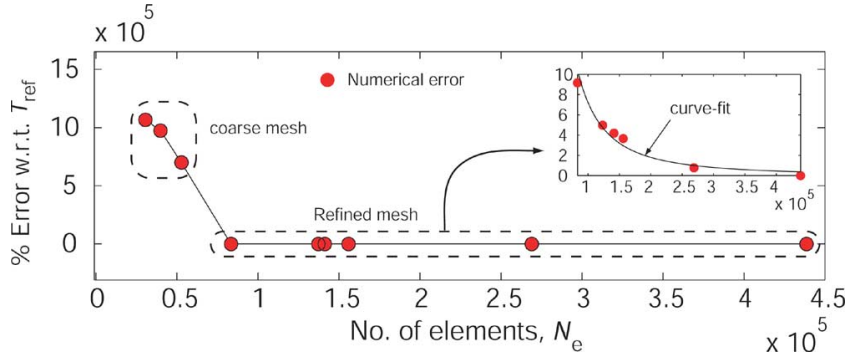

Fig. 8. Variation of percentage error in calculating $T_{\max }$ with the number of elements $N_{e}$ (here, $T_{\text {ref }}=1.03 \times 10^{-13} \mathrm{~N}$-m is the converged value of $T_{\max }$ corresponding to 438685 elements).

is the number of perforations, and the plate area $A_{\text {plate }}=$ $400 \mu \mathrm{m} \times 400 \mu \mathrm{m}$, we get $P_{r}=0.0095 \ll 0.1$. Moreover, the ratio of the perforation size to the air-gap thickness $P_{h}=l_{h} / h_{0}=0.037$ is also very small. Since the perforation size is very small compared to the pitch of the perforation distribution and the air gap thickness, we neglect the effect of perforations in the modeling.

\section{A. Validation of Numerical Procedure}

To validate the numerical procedure, we compare the numerically computed damping constant with the analytical value obtained from (4) for $L=400 \mu \mathrm{m}, W=400 \mu \mathrm{m}$, and $h_{0}=$ 10 to $80 \mu \mathrm{m}$. Air is used as the surrounding medium. The density and viscosity of air are taken to be $1.2 \mathrm{~kg} / \mathrm{m}^{3}$ and $1.8 \times$ $10^{-5} \mathrm{~N}-\mathrm{s} / \mathrm{m}^{2}$, respectively. While the excitation frequency is $529.2 \mathrm{~Hz}$, the amplitude of angular displacement is taken as $0.004 \mathrm{rad}$, which corresponds to $1 \%$ of the maximum allowable angular displacement when the tip of the electrode touches the substrate. The numerical damping constant is obtained by solving the Navier-Stokes equation in 3-D domain with the trivial boundary condition on the plate edges as explained in the previous section. It is found that the analytical results compare very well with the numerical result for $h_{0} / L \leq 0.1$ with less than $10 \%$ error as shown in Fig. 6. Beyond 40- $\mu$ m gap thickness, the effect of large air gap increases due to the nonuniform pressure variation across the gap thickness. At $80 \mu \mathrm{m}$, the analytical formula gives an error of about $33 \%$. Thus, we validate our numerical procedure that matches well with the analytical result for small air gap to length ratios. Subsequently, we do further comparisons with the experimental results under different boundary conditions in the next section.

Another important aspect in numerical modeling is the selection of the oscillation amplitude, which should be comparable to the experimental values. The experimental value of the oscillation amplitude is $0.035 \mathrm{rad}$ at $529.2 \mathrm{~Hz}$ (see Fig. 5), which is about $8.75 \%$ of the maximum allowable angular amplitude $(\approx 0.4 \mathrm{rad}$ when the tip of the electrode touches the substrate). In numerical simulations, we vary the oscillation amplitude from $1 \%(0.004 \mathrm{rad})$ to $10 \%(0.04 \mathrm{rad})$ of the maximum allowable angular displacement and compute the corresponding damping constant. In this range, we find the damping constant to be independent of the amplitude as expected (for small amplitude motion) with a variation as small as $0.1 \%$. Based on these results, we select $0.004 \mathrm{rad}$ as the amplitude of angular 


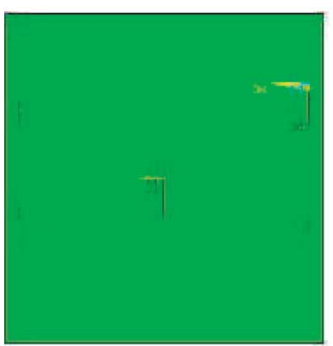

(a)

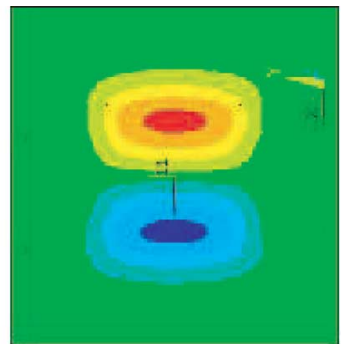

(b)

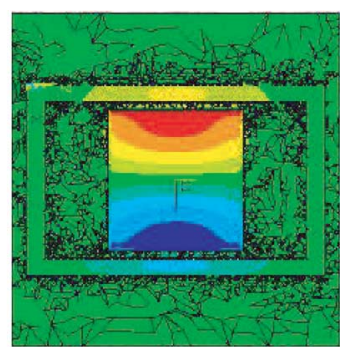

(c)

Fig. 9. (a) Pressure distribution on the top surface of the fluid domain of Fig. 7(b) (here, the pressure variation is found to be zero). (b) Pressure distribution on the bottom surface. (c) Pressure distribution on the oscillating plate and the inner frame in the domain bounded by the bottom surface and section A- $\mathrm{A}^{\prime}$ shown in Fig. 7(b) (black marks around the structure represent the edges of FLUID142 elements cut by the section A-A').

displacement for all simulations in Section III-B, which is $1 \%$ of the maximum allowable angular displacement $(0.4 \mathrm{rad})$.

\section{B. Comparison of Numerical, Experimental, and Analytical Results}

By following the numerical procedure outlined earlier, we calculate the quality factor of the MEMS torsion mirror. The mirror is subjected to an angular displacement $\phi=\Phi \sin 2 \pi f t$, where $f=529.2 \mathrm{~Hz}$ is the damped natural frequency at atmospheric pressure as found from the experimental result shown in Fig. 5, and $\Phi=0.004$ rad is the amplitude of angular displacement, which is taken as 0.01 times the maximum possible angular displacement (when the plate touches the bottom electrode at the far end). Since the bottom electrode thickness $(0.3 \mu \mathrm{m})$ and the static angular displacement $\left(5.0 \times 10^{-3} \mathrm{rad}\right)$ due to the bias voltage are very small, we neglect their effects on the nominal gap thickness $h_{0}$. From the numerical results, we find that for the present structure, the perforations in the plate can be neglected without any significant loss in accuracy in calculating the total reactive torque and the quality factor. The percentage error introduced by neglecting perforations is $\sim 0.087 \%$ and $\sim 0.79 \%$ in the evaluation of total torque and quality factor, respectively. This is largely because the perforation size $(3 \mu \mathrm{m})$ is very small compared to the pitch $(\sim 32.5 \mu \mathrm{m})$ and air gap thickness $(80 \mu \mathrm{m})$ [22], [23]. Therefore, in the subsequent discussions, we neglect the perforations in the modeling.

1) Comparison of Numerical and Experimental Results: To validate the numerical procedure, we first create a 3-D fluid domain around the torsional mirror of the given dimensions (see Table I). The lateral boundaries of the fluid domain are fixed at $l_{p} / 2$ from the longer external edge and $l_{p} / 10$ from the shorter external edge of the inner frame, and the top is fixed at $3 h_{0} / 4$ from the top of the movable plate (see Fig. 7). The extension is obtained by gradually moving the boundary outwards until the obtained solution converges for the pressure distribution around the structure. The fluid domain is meshed with tetrahedral FLUID 142 elements of ANSYS. The zero-pressure boundary condition is applied on the extended surfaces; zero velocity condition is applied on the bottom surface and the surfaces around the static inner frame; and the sinusoidal angular velocity is applied to the surfaces binding the moving structure. Transient response calculations are carried out for six cycles because six cycles are found to be sufficient to attain steady state. On the time axis, 30 points are used per cycle. At each time step, local iterations are done to achieve convergence of order five [i.e., $\left(P_{i+1}-P_{i}\right) / P_{i} \leq 10^{-5}$, where $i$ denotes the local iteration step] in the calculation of pressure variation. The under-relaxation factors for iterating velocity and pressure are taken as 0.5 to ensure a fast and smooth convergence [24]. After calculating the total reactive torque and subsequently breaking it into its damping and spring components, we calculate the quality factor from (9). To ensure the accuracy of the numerical method, we calculate the maximum torque $T_{\max }$ around the oscillating structure for different finite-element discretizations, which are used to mesh the fluid domain around the structure as shown in Fig. 7. Convergence of the finite-element calculations is clearly seen by plotting the percentage error against the number of elements (Fig. 8), where the error is computed with respect to the converged value of $T_{\max }$. If $N_{e}$ is the number of elements, then the percentage error seems to reduce as $1 / N_{e}^{2}$ (see inset in Fig. 8). The numerical error is below $1 \%$ if the number of elements is over 250000 . Fig. 9 shows the pressure distribution on the top surface, the bottom surface, and the section $\mathrm{A}-\mathrm{A}^{\prime}$ [see Fig. 7(b)] along the bottom surface of the oscillating structure in the fluid domain, which is meshed with 438685 elements. The converged values of the maximum torque $T_{\max }$ and the damping torque $T_{d 0}$ are $1.03 \times 10^{-13} \mathrm{~N}-\mathrm{m}$ and $1.0 \times 10^{-13} \mathrm{~N}-\mathrm{m}(=100 \mu \mathrm{N}-\mathrm{nm})$, respectively, which correspond to the angular oscillation of $\phi=0.004 \sin (3325.10 t)$. Finally, we get $Q_{\text {num }}=9.27$, which is very close to the experimentally determined quality factor $Q_{\exp }=9.19$, giving an error of about $0.87 \%$.

The effect of squeeze-film flow between the vibrating plate and the fixed plate, and the effect of air flow through the side walls and the top surface of the vibrating plate, are compared and analyzed for different boundary conditions, as described in Fig. 10. In case (a), the zero-pressure boundary condition is taken on the edges of the vibrating structure where only squeeze-film damping is considered. In case (b), the zeropressure boundary condition is applied on the extended boundary so as to include the open border effect and the effect of the side walls on fluid flow (although the effect of the side wall is negligible in the present case). In case (c), the effect of the side and top surfaces on fluid flow is taken by applying the zero-pressure boundary condition on the surfaces extended from the side and top surfaces of the vibrating plate. Finally, 


\begin{tabular}{|c|c|c|c|}
\hline S. No. & Boundary Condition & Pressure Pattern & Quality Factor \\
\hline (a) & & & 27.9 \\
\hline (b) & & & 14.35 \\
\hline (c) & $\square$ & & 10.16 \\
\hline (d) & $\square \Longleftrightarrow$ & & 9.27 \\
\hline \multicolumn{4}{|c|}{$\mathrm{s}$} \\
\hline & $\begin{array}{l}\text { MIN } \\
(-v e)\end{array}$ & & $\begin{array}{l}\text { IAX } \\
\text { vive) }\end{array}$ \\
\hline
\end{tabular}

Fig. 10. Patterns of average pressure variation and quality factor of a 3-D structure executing torsional motion under open and partially blocked boundary conditions. Different boundary conditions in (a)-(d) are used to capture different effects as mentioned in Fig. 1(c)-(f). Here, trivial pressure boundary condition is applied on the dotted lines, zero velocity condition is applied on the lines around the blank rectangle, and sinusoidal velocity is applied on the lines around the grey-colored rectangle. Here, the movement of mesh is scaled-up for better view.

TABLE II

Percentage Error $\left(Q_{\text {num }}-Q_{\text {exp }} / Q_{\exp } \times 100\right)$ OBtAined UNDER DIFFERENT BOUNDARY CONDITIONS WiTH RESPECT TO EXPERIMENTAL VALUE OF QUALITY FACTOR $Q_{\exp }=9.19$ FOR THE STRUCTURE SHOWN IN FIG. 1

\begin{tabular}{|c|c|c|c|}
\hline \hline Case & Boundary Condition & $Q_{\text {num }}$ & $\%$ error \\
\hline \hline 1. & $\begin{array}{c}\text { Squeeze effect (Sq) } \\
\text { in fig. 10(a) }\end{array}$ & $Q_{\mathrm{Sq}}=27.9$ & 203.59 \\
\hline 2. & $\begin{array}{c}\text { Squeeze(Sq)+Side(S) } \\
\text { effect in fig. 10(b) }\end{array}$ & $Q_{\mathrm{Sq}+\mathrm{S}}=14.35$ & 56.14 \\
\hline 3. & $\begin{array}{c}\text { Squeeze(Sq)+Side(S) } \\
+\mathrm{Top}(\mathrm{T}) \text { effect in } \\
\text { fig. 10(c) }\end{array}$ & $Q_{\mathrm{Sq}+\mathrm{S}+\mathrm{T}}=10.16$ & 10.55 \\
\hline 4. & $\begin{array}{c}\text { Squeeze(Sq)+Side(S) } \\
+ \text { Top(T) effect with static } \\
\text { outer frame in fig. 10(d) }\end{array}$ & $Q_{\mathrm{Sq}+\mathrm{S}+\mathrm{T}}=9.27$ & 0.87 \\
\hline \multicolumn{2}{|c|}{} & \\
\hline
\end{tabular}

we imitate the actual boundary conditions that exist around the MEMS device (shown in Fig. 3) in case (d) by putting a static frame near the boundaries of the vibrating plate. In this case, the free flow of the fluid from the boundaries of the vibrating plate gets partially obstructed by the presence of the nearby static structure.

In Table II, the quality factor calculated numerically for the aforementioned cases is compared with the experimental value of the quality factor $Q_{\exp }=9.19$ for the device shown in Fig. 3 . It is found that the condition in case 1, based on which most of the compact formulas are derived, gives an error of about $203 \%$, while the case 3 that considers the effect of the side and top surfaces (i.e., fully open boundary condition) of the vibrating structure reduces the error to about $11 \%$. However, when the actual condition is considered, the error reduces even further and gives a value of about $1 \%$ only.
TABLE III

ERror ObTained B Y USING CONVENTIONAL ANALYTICAL SOLUTION WiTH OR WithOUT OPEN BORDER EFFECT FOR THE GIVEN STRUCTURE. HERE, error $=\left|Q_{\text {anal }}-Q_{\exp } / Q_{\exp }\right| \times 100$

\begin{tabular}{|c|c|c|c|}
\hline \hline S.No. & Case & Quality Factor, $Q$ & $\%$ error \\
\hline \hline 1. & $\begin{array}{c}\text { Squeeze film without } \\
\text { considering border effect. } \\
\text { Analytical result based on eqn. (4) }\end{array}$ & $Q_{\text {exp }}=9.19$ & 356 \\
\hline 2. & $\begin{array}{c}\text { Squeeze film with correction } \\
\text { for the border effect. } \\
\text { Analytical result based on eqn. (4) } \\
\text { with the elongation of } 1.3 h_{0}[10]\end{array}$ & $Q_{\text {exp }}=9.19$ & 14 \\
\hline
\end{tabular}

Although the boundary condition considered in case (d) is specific to the present MEMS device, it represents a more generic case of partially blocked boundaries. It is clear from the results that such boundaries need to be modeled carefully for reasonably accurate results.

2) Comparison of Analytical Models With Experimental Results: In Table III, the experimental result is compared with the analytical solution obtained by solving the conventional Reynolds equation [15] with zero-pressure boundary condition on the boundaries of the oscillating plate [4]. The relevant dimensions are taken from Table I.

In case 1 of Table III, the zero-pressure boundary condition is applied on the original boundary of the oscillating inner plate, as shown in case (a) of Fig. 10, thereby ignoring the open border effect. The analytical quality factor is calculated using (4) and (5). It is found that the analytical result gives an error of about $356 \%$ when compared with the experimental result. The error in this case is greater than that in case 1 of Table II. This is so because in addition to the error shown 


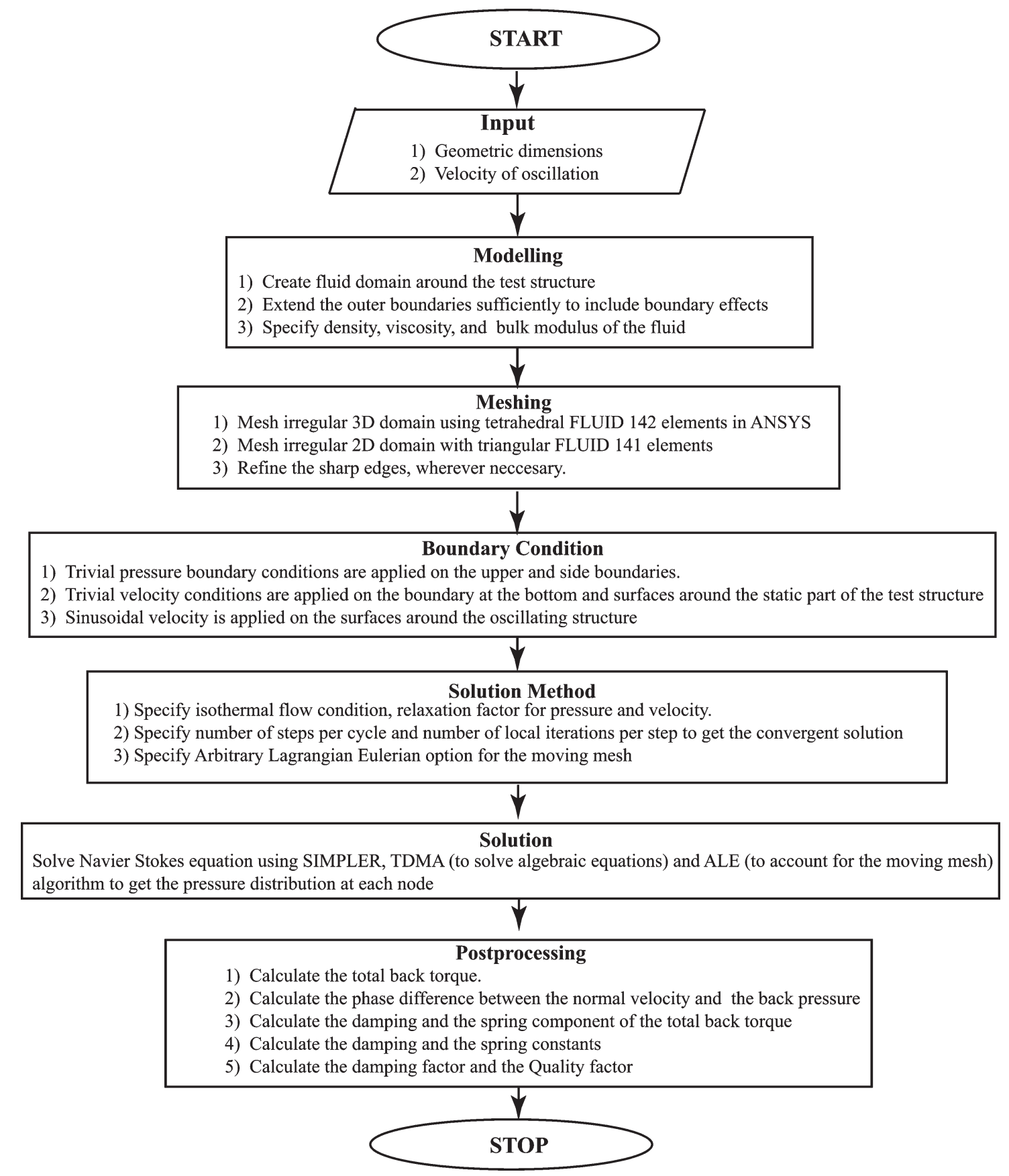

Fig. 11. Algorithm to calculate the quality factor numerically.

in Table II, the error due to the violation of one of the most basic assumptions-very small air-gap-to-length ratio used in deriving the Reynolds equation-also exists in this case, i.e., the 2-D flow assumption of Reynolds equation is violated.

In case 2, the zero-pressure boundary condition is applied on the boundary of the surface extended by $(1.3 / 2) h_{0}[10]$ to account for the fully open boundary effect. The analytical quality factor is then calculated using (4) and (5). Here, the effective dimensions are calculated by $L_{\text {eff }}=L+1.3 h_{0}$ and $W_{\text {eff }}=W+1.3 h_{0}$, whereas the moment of inertia $I=$ $2.106 \times 10^{17} \mathrm{~kg}-\mathrm{m}^{2}$ is calculated using the original dimensions of the structure and $\omega=3.325 \times 10^{3} \mathrm{rad} / \mathrm{s}$ from the experimental result. The analytically computed result differs from the experimentally measured value by $14 \%$, which is probably due to the fact that the factor $1.3 h_{0}$ does not account for the partially blocked boundaries such as the case in the given structure. Since the extension factor given by Veijola [10] accounts for fully open boundary conditions, $Q_{\text {anal }}$ matches well with the corresponding $Q_{\text {num }}$ in case 3 of Table II with an error of about 3\%. It also validates our computational result.

Finally, from the comparative studies of the experimental, numerical, and analytical results, we find that the extension factor $1.3 h_{0}$ is indeed useful to capture border effects of fully open boundaries. To the best of our knowledge, this is the first experimental verification of this factor. 


\section{CONCLUSION}

CFD is suitable for studying the various effects of different boundary conditions such as fully open, fully closed, and partially closed boundaries in oscillating MEMS devices. We also use it to study a problem where the air gap to plate length ratio is large, which makes the conventional Reynolds equation inapplicable. We take a fairly complex torsional MEMS structure with large air gap and complicated boundary conditions, and simulate the effect of squeeze-film damping by progressively refining the model for the boundary conditions. We compare the simulation results with experimentally obtained results (captured by the $Q$-factor of the device) and show how the numerically computed results get progressively close to the experimental value with refinements in the boundary condition modeling. Starting from the most common and naive model of the trivial pressure boundary condition on the fluid domain enclosed by the oscillating structure, which gives an error of $203 \%$ compared to the experimental value, we get to the most realistic model of the partially blocked boundary that gives results within $1 \%$ of the experimental value. We then compare the numerical as well as the experimental result with relevant analytical models and show that Veijola's analytical model with an appropriate correction for open border effects comes closest with an error of $14 \%$.

\section{APPENDIX}

The numerical procedure used to calculate the quality factor due to fluid damping is given in Fig. 11.

\section{REFERENCES}

[1] H. Hosaka, K. Itao, and S. Kuroda, "Damping characteristics of beam-shaped micro-oscillators," Sens. Actuators A, Phys., vol. 49, no. 9 , pp. 87-95, 1995.

[2] J. Yang, T. Ono, and M. Esashi, "Energy dissipation in submicrometer thick single-crystal silicon cantilevers," J. Microelectromech. Syst., vol. 11, no. 6, pp. 775-783, Dec. 2002.

[3] J. J. Blech, "On isothermal squeeze films," J. Lubr. Technol., vol. 105, pp. 615-620, 1983.

[4] F. Pan, J. Kubby, E. Peeters, A. T. Tran, and S. Mukherjee, "Squeeze film damping effect on the dynamic response of a MEMS torsion mirror," J. Micromech. Microeng., vol. 8, no. 3, pp. 200-208, 1998.

[5] Y.-J. Yang and S. D. Senturia, "Numerical simulation of compressible squeezed-film damping," in Proc. Solid-State Sensor and Actuator Workshop, Jun. 1996, pp. 76-79.

[6] Y.-J. Yang, M. A. Gretillat, and S. D. Senturia, "Effect of air damping on the dynamics of nonuniform deformations of microstructures," in Proc. Transducers, Int. Conf. Solid-State Sens. Actuators, 1997, vol. 2, pp. 1093-1096.

[7] E. S. Hung, Y.-J. Yang, and S. D. Senturia, "Low-order models for fast dynamical simulation of MEMS microstructures," in Proc. Transducers, Int. Conf. Solid-State Sens. Actuators, 1997, vol. 2, pp. 1101-1104.

[8] E. S. Hung and S. D. Senturia, "Generating efficient dynamical models for microelectromechanical systems from a few finite-element simulation runs," J. Microelectromech. Syst., vol. 8, no. 3, pp. 280-289, Sep. 1999.

[9] S. Vemuri, G. K. Fedder, and T. Mukherjee, "Low-order squeeze film model for simulation of MEMS devices," in Proc. 3rd Int. Conf. Model. and Simul. Microsyst., 2000, pp. 205-208.

[10] T. Veijola, A. Pursula, and P. Ráback, "Extending the validity of squeezedfilm damper models with elongations of surface dimensions," J. Micromech. Microeng., vol. 15, no. 9, pp. 1624-1636, Sep. 2005.

[11] Z. Hao, R. Clark, J. Hammer, M. Whitley, and B. Wingfield, "Modeling air-damping effect in a bulk micromachined 2D tilt mirror," Sens. Actuators A, Phys., vol. 102, no. 1, pp. 42-48, Dec. 2002.

[12] R. B. Darling, C. Hivick, and J. Xu, "Compact analytical modelling of squeeze film damping with arbitrary venting conditions using a Green's function approach," Sens. Actuators A, Phys., vol. 70, no. 1/2, pp. 32-41, 1998.

[13] ANSYS 9, Finite Element Solver for Multiphysics Problems. [Online]. Available: http://www.ansys.com/

[14] G. K. Batchelor, An Introduction to Fluid Dynamics. Cambridge, U.K.: Cambridge Univ. Press, 1997.

[15] O. Pinkus and B. Sternlicht, Theory of Hydrodynamic Lubrication. New York: McGraw-Hill, 1961.

[16] W.-L. Li, "Analytical modelling of ultra-thin gas squeeze film," Nanotechnology, vol. 10, no. 4, pp. 440-446, 1999.

[17] T. Veijola, K. Ruokonen, and I. Tittonen, "Compact squeezed-film damping model including the open border effects," in Proc. MSM, 2001, pp. 76-79.

[18] L. E. Kinsler, A. R. Frey, A. B. Coppens, and J. V. Sanders, Fundamentals of Acoustics. New York: Wiley, 2000.

[19] T. Veijola, "Acoustic impedance elements modeling oscillating gas flow in micro channels," in Proc. MSM, 2001, pp. 96-99.

[20] S. S. Rao, Mechanical Vibration. New York: Wesley, 1995.

[21] G. A. Bird, Molecular Gas Dynamics and the Direct Simulation of Gas Flows. Oxford, U.K.: Oxford Univ. Press, 1996.

[22] M. Bao, H. Yang, Y. Sun, and P. J. French, "Modified Reynolds equation and analytical analysis of squeeze film air damping of perforated structures," J. Micromech. Microeng., vol. 13, no. 6, pp. 795-800, Nov. 2003.

[23] S. S. Mohite, K. Haneesh, V. R. Sonti, and R. Pratap, "Analytical solutions for the stiffness and damping coefficients of squeeze films in MEMS devices with perforated back plates," J. Micromech. Microeng., vol. 15, no. 11, pp. 2083-2092, Nov. 2005.

[24] S. V. Patankar, Numerical Heat Transfer and Fluid Flow. New York: Hemisphere, 1980.

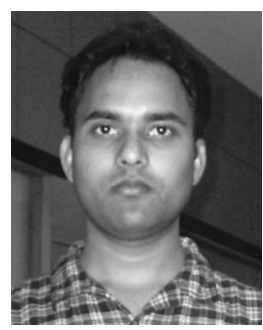

Ashok Kumar Pandey received the B.E. degree in mechanical engineering from Bhilai Institute of Technology, Durg, India, in 2001, and the M.S. degree from the Indian Institute of Science, Bangalore, India, in 2003. He is currently working toward the $\mathrm{Ph} . \mathrm{D}$. degree in MEMS at the Indian Institute of Science.

His current research focus is on the investigation of different energy dissipation mechanisms in micro and nano devices.

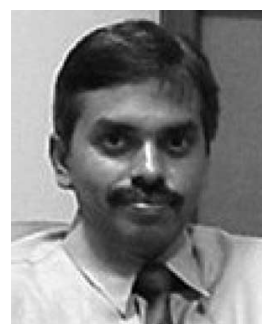

Rudra Pratap (M'07) received the B.Tech. degree from the Indian Institute of Technology (IIT), Kharagpur, India, in 1985, the M.S. degree in mechanics from the University of Arizona, Tucson, in 1987, and the Ph.D. degree in theoretical and applied mechanics from Cornell University, Ithaca, NY, in 1993.

From 1993 to 1996, he was with the Sibley School of Mechanical and Aerospace Engineering, Cornell University. In 1996, he was with the Indian Institute of Science, Bangalore, India. From 1997 to 2000, he was an Adjunct Assistant Professor at Cornell University. He is currently an Associate Professor in the Department of Mechanical Engineering, Indian Institute of Science. His research interests are MEMS design, computational mechanics, nonlinear dynamics, structural vibration, and vibroacoustics.

Dr. Pratap is a member of the Institute of Smart Structure and System and is on the Editorial Board of the Journal of Computers, Materials and Continua (CMC).

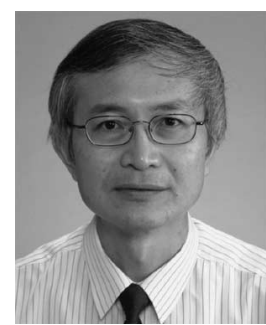

Fook Siong Chau received the Ph.D. degree from Nottingham University, Nottingham, U.K.

After working as a Research Scientist and Project Engineer in a research laboratory, in 1981, he joined the National University of Singapore, Singapore, where he is currently an Associate Professor in the Department of Mechanical Engineering. He also heads the Applied Mechanics Academic Group. His main research interests are in the modeling, simulation, and characterization of microsystems, particularly MOEMS, and the development and application of optical techniques for nondestructive evaluation of components.

Dr. Chau is a member of the Institution of Engineers, Australia, and the Institution of Engineers, Singapore. 